\title{
Increased cerebrospinal fluid flow through the foramen of Magendie after decompression for Chiari I malformation
}
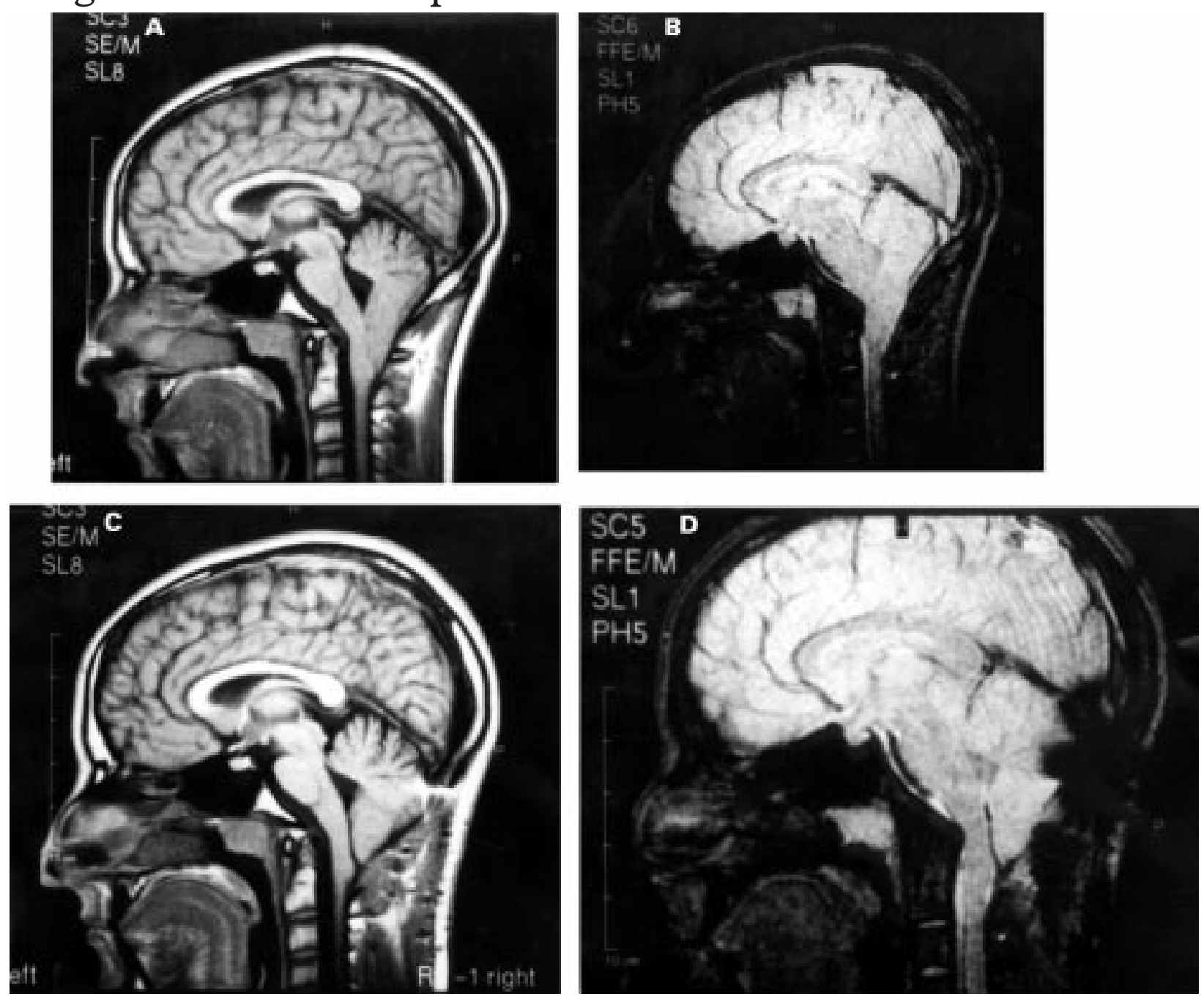

A 15 year old patient with a 6 month history of cough headache was diagnosed as having a Chiari I malformation. The midsagittal T1 weighted MRI shows the tonsillar ectopia (figure A). A flow sensitive MRI, cardiac triggered gradient echo $\mathrm{T}^{\star}{ }^{\star}$ weighted sequence obtained at $200 \mathrm{~ms}$ from the $\mathrm{R}$ wave, showed absence of CSF flow through the foramen of Magendie (figure B). After craniovertebral decompression leaving the arachnoid intact, using the technique described by Sahuquillo et al, ${ }^{1}$ the characteristic pattern of headache disappeared without relapsing symptoms after a 2 year follow up. Postoperative MRI shows a patent communication between the fourth ventricle and the cisterna magna (figure C). The flow sensitive MR sequence shows a low signal tract from the fourth ventricle to the cisterna magna indicating rapidly flowing CSF during the early vascular systole (figure D). In this image, the low signal appreciated in the inion area represents an artifact from metallic shaving due to drilling.

Clinical improvement together with MR findings consistent with restoration of CSF dynamics link cough headache in the Chiari I malformation to impedance of caudal CSF flow through the foramen of Magendie. Restoring CSF dynamics should be the main goal in the surgical treatment of patients with the Chiari I malformation presenting with exertional symptoms. Extra-arachnoid techniques achieve this, not only by providing more room in the subarachnoid space in the foramen magnum region and by breaking the craniospinal dissociation mechanism, but also by facilitating egress of CSF through the foramen of Magendie.

DAVID SANTAMARTA MARIA ELENA KUSAK JOSE MARIA DE CAMPOS

Department of Neurosurgery, Hospital del Rio Hortega, Valladolid, Spain

JOSE MARIA SIERRA Centro de Diagnostico, Valladolid, Spain

Correspondence to: Dr D Santamarta, Servicio de Neurocirugia, Hospital Universitario de Salamanca, Pasco de San Vicente, 58-182, 37007 Salamanca, Spain.

1 Sahuquillo J, Rubio E, Poca MA, et al. Posterior fossa reconstruction: a surgical technique for the treatment of Chiari I malformation and Chiari $1 /$ syringomyelia complex: preliminary results and magnetic resonance imaging quantitative assessment of hindbrain migration. Neurosurgery 1994;35:874-85. 